Notfall Rettungsmed 2013 · 16:549-555

DOI 10.1007/s10049-013-1764-0

Online publiziert: 23. Oktober 2013

(c) The Authors 2013. This article is published

with open access at link.springer.com

A. Donner ${ }^{1} \cdot$ C. Adler ${ }^{2}$

${ }^{1}$ Deutsches Zentrum für Luft- und Raumfahrt e.V., Institut für

Kommunikation und Navigation, Oberpfaffenhofen, Weßling

${ }^{2}$ Ludwig-Maximilians-Universität München, Department Psychologie,

Klinische Psychologie und Psychotherapie, München

\title{
Patientenregistrierung im Massenanfall
}

\section{Zukünftige Anforderungen an ein elektronisches Dokumentationssystem}

Die Forschungsprojekte A.L.A.R.M., e-Triage, MANET, SOGRO und SPIDER ${ }^{1}$ sowie das Forschungsprojekt UCSE ${ }^{2}$ führten in den vergangenen Jahren Untersuchungen durch, inwiefern EDV-Systeme Einsatzkräfte bei einem MANV unterstützen können. Auch wenn sich die technischen Umsetzungen der Projekte im Detail unterscheiden, sind dennoch die erarbeiteten Anforderungen an entsprechende elektronische Systeme nahezu deckungsgleich. Der nachfolgende Text fasst wesentliche Ergebnisse zusammen und beschreibt den identifizierten Handlungsbedarf.

\section{Status quo}

Ein Massenanfall von Verletzten (MANV) ist laut Referenz [1]

ein Notfall mit einer größeren Anzahl von Verletzten oder Erkrankten sowie anderen

\footnotetext{
1 Vom Bundesministerium für Bildung und Forschung geförderte Forschungsprojekte im Themenfeld "Schutz und Rettung von Menschen" der Programmlinie "Szenarienorientierte Sicherheitsforschung" des Sicherheitsforschungsprogramms. Projektträger ist die VDI Technologiezentrum $\mathrm{GmbH}$.

2 User Centered Systems for Emergency: Durch das Förderprogramm Hochschule-Wirtschaft-Transfer (HWT III) der Innovationsstiftung Schleswig-Holstein und die Behra Unternehmensberatung $\mathrm{GmbH}$ gefördertes Projekt zur mobilen elektronischen Datenerfassung bei einem MANV.
}

Geschädigten oder Betroffenen, der mit der vorhandenen und einsetzbaren Vorhaltung des Rettungsdienstes aus dem Rettungsdienstbereich nicht bewältigt werden kann.

Ein MANV lässt sich deswegen nicht unbedingt durch die absolute Anzahl von Verletzten und Betroffenen definieren, sondern ist die Folge eines relativen Missverhältnisses zwischen der Anzahl der zu behandelnden Patienten und der zur Verfügung stehenden Rettungsdienstressourcen und Krankenhauskapazitäten. Dementsprechend werden weitere als Reserve vorgehaltene Kräfte (Schnell-Einsatz-Gruppen) und - falls nötig - überregionale Hilfe nachalarmiert. Hauptunterschied zur normalen Notfallrettung ist die Einrichtung einer erweiterten Führungsstruktur vor Ort (Einsatzleitung bestehend aus organisatorischem Leiter Rettungsdienst und leitendem Notarzt sowie weiteren unterstützenden Kräften), die alle Einsatzkräfte und (Transport-)Maßnahmen koordiniert.

Sichtung (Triage; [2]) bezeichnet die ethisch schwierige Aufgabe, eine Entscheidung darüber zu treffen, wie die bei einem unerwartet auftretenden Schadensereignis knappen, personellen und materiellen Mittel aufzuteilen sind, um die Versorgung einer größtmöglichen Anzahl von verletzten und/oder erkrankten Personen zu gewährleisten. Die Diskrepanz zwischen vorhandenen Ressourcen und notwendigen Maßnahmen erfordert eine Priorisierung, deren Ziel es ist, 


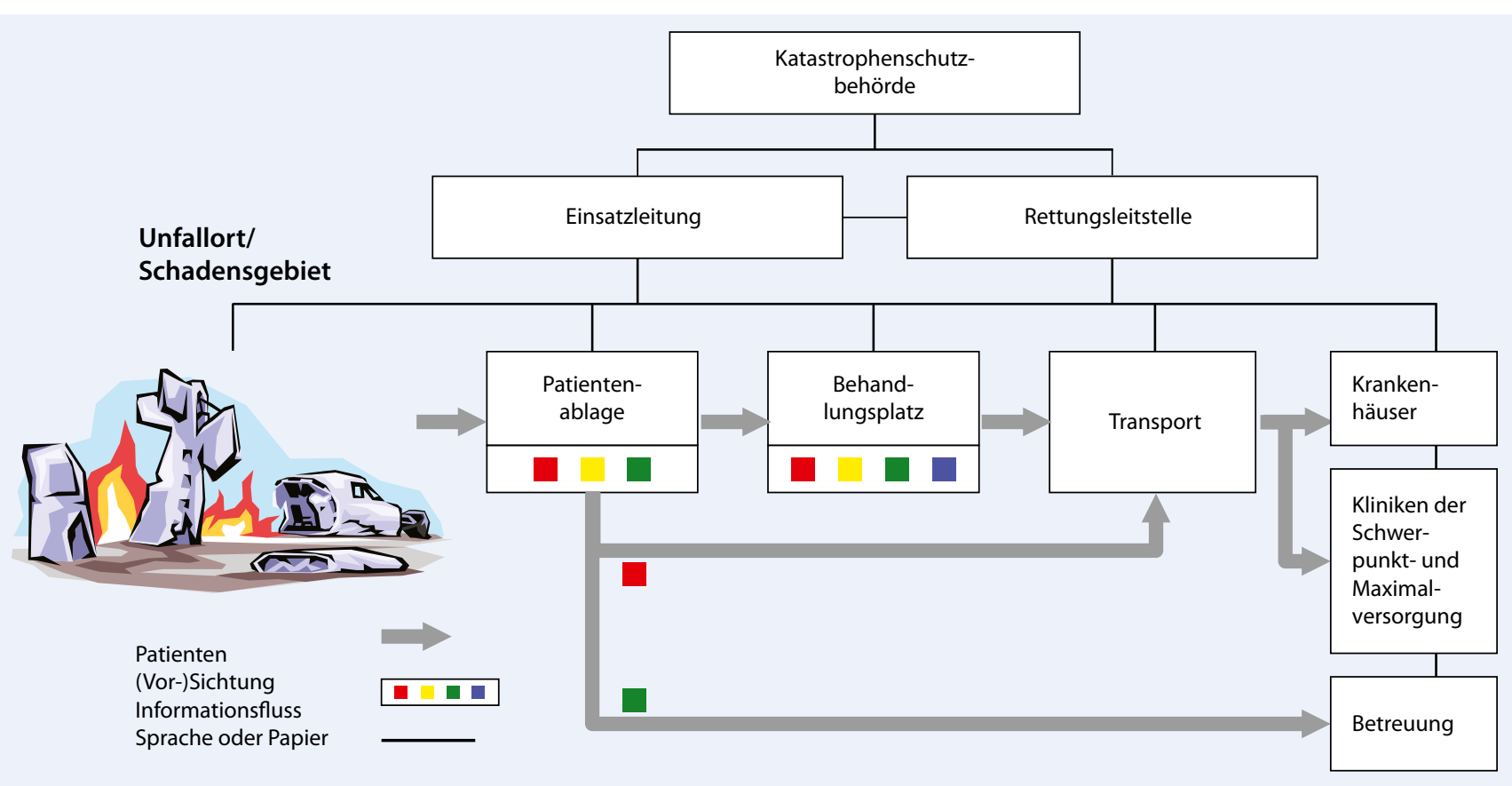

Abb. 1 A Gängiges theoretisches Patientenversorgungsschema MANV. (Quelle: http://e-triage.de/; 02.08.2013, mit freundl. Genehmigung A. Donner)

akut lebensbedrohlich verletzte Personen zu identifizieren, die einer sofortigen Behandlung oder eines schnellen Transports bedürfen, und sie von denjenigen Betroffenen zu trennen, die nicht oder nur leicht bzw. gering verletzt sind [3].

\section{Sichtung und Registrierung - Voraussetzung für die Arbeit der Einsatzleitung}

Sichtung und Registrierung vor Ort und/ oder an einer Patientenablage ermöglichen einen Überblick über die Anzahl der Verletzten, den Schweregrad der Verletzungen und die Behandlungsdringlichkeit, um die Patienten schnellstmöglich einer adäquaten medizinischen Versorgung zuzuführen. Wie in • Abb. 1 dargestellt, erfolgt eine Priorisierung der Patienten entlang der weiteren Stationen der Versorgungskette gemäß ihrer Dringlichkeit. Dieser Ablauf ist als theoretischer und idealisierter Maximalausbau der Patientenversorgungskette zu verstehen. Tatsächlich ist die Darstellung eine (beinahe unzulässige) Abstrahierung, da die zeitliche und räumliche Dimension des Einsatzgeschehens nicht berücksichtigt wird. Aufgrund von Alarmierungsund Anfahrtszeiten können die einzelnen Strukturen nicht zeitgleich erstellt werden und es gibt nur in den seltensten Fällen definierte Übergabepunkte zwischen den Stationen. Die Entscheidung, ob ein Behandlungsplatz eingerichtet werden soll, wird in Deutschland unterschiedlich diskutiert bzw. obliegt in letzter Instanz dem zuständigen Einsatzleiter.

\section{Bestehendes Konzept der Patientenanhängekarten - einige entscheidende Schwachstellen}

Bei der Verwendung von Patientenanhängekarten bleiben die Daten über die $\mathrm{Pa}$ tienten an den Patienten, und die von den Sichtungsteams geführten Listen stehen letztendlich erst nach Abschluss der Sichtungsarbeiten zur Verfügung. Anschließend können diese Listen nur durch Abschreiben bzw. Diktat am Funk zusammengeführt werden. Eine Einsatzleitung (und somit auch die Leitstelle) sieht deswegen immer ein stark verzögertes Lagebild und muss deswegen auf der Grundlage von sich bereits überholenden, unvollständigen oder partiell (mitunter auch gänzlich) falsch übermittelten Daten Entscheidungen treffen.

Die Reihenfolge, in der die Patienten mit der höchsten Transportkategorie abtransportiert werden, legt im Normalfall der leitende Notarzt fest, was al- lerdings zumindest annähernde Kenntnis von der Anzahl an Patienten, deren Sichtungskategorie, Verletzungsart sowie Versorgungs- und Transportkapazität voraussetzt. Die Verteilungskommunikation mit den aufnehmenden Krankenhäusern erfolgt - wenn überhaupt - fast ausschließlich über die Leitstelle. Es ist naheliegend, dass diese individuellen Abfragen bei einer zu großen Anzahl von Notfallpatienten nicht mehr durchführbar sind und dann auf vorbereitete Verteilungspläne zurückgegriffen werden muss [4].

Anzumerken ist, dass derzeit in den einzelnen Stationen der Versorgungskette mehrere Patientenverwaltungslisten parallel geführt werden, die alle aufwändig händisch gepflegt und miteinander abgeglichen werden müssen. Zudem verwenden oftmals verschiedene Rettungsorganisationen unterschiedliche Dokumentationssysteme, die über die Landkreisgrenzen oder Rettungsleitstellenbereiche hinaus nicht kompatibel sind. Schätzungen zufolge gibt es beispielsweise in Deutschland ca. 15 verschiedene Arten von Patientenanhängekarten die sich in Form, Inhalt und Anwendung teils deutlich unterscheiden [4]. Hinzu kommen Dutzende von Vorgehensweisen für die Kennzeichnung der Karten mit eindeutigen Nummern sowie regional unterschiedliche Vorgaben, 
welche Sichtungskategorien zu verwenden und wie sie zu vergeben sind.

Eine bundesweite Harmonisierung erscheint zwingend notwendig, da insbesondere bei Großschadenslagen ein hohes Maß an Effizienz erforderlich ist, die überregionale Hilfe im Vordergrund steht und deshalb Verfahren und technische Hilfsmittel kompatibel sein müssen.

\section{Erhebung von Patientendaten bei einem MANV}

In - Abb. 2 wird exemplarisch gezeigt, welche Patientendaten entlang der Versorgungskette bei einem MANV erhoben werden können [5]. Nach der ersten Sichtung (meistens Vorsichtung) haben die Patienten mindestens eine eindeutige Kennzeichnung (Identifier, ID) und eine Sichtungskategorie [6]. Ab Behandlungsplatz bzw. beim Transport werden insbesondere therapeutische Maßnahmen (z. B. verabreichte Medikamente), aber auch bei ausreichend verfügbaren personellen Ressourcen die Identitäten der Betroffenen dokumentiert.

Das Rettungsdienstprotokoll und das Notarzteinsatzprotokoll („DIVI-Protokoll“) werden bereits zunehmend elektronisch erfasst, da sich Forderungen aus der Qualitätssicherung (z. B. einheitliche Dokumentation) leichter abbilden lassen als mit Papierformularen und als Nebeneffekt die Abrechnung vereinfacht wird. $\mathrm{Zu}$ letzt wurde im September 2011 der Minimale Notarztdatensatz der Deutschen Interdisziplinären Vereinigung für Intensivund Notfallmedizin (DIVI) „MIND3“ veröffentlicht [7], für den es bereits (nicht einheitliche) Softwareimplementierungen gibt. Des Weiteren sind oftmals elektronische Systeme im Rettungsdienst/Krankentransport für das Flottenmanagement in Verwendung. Diese Systeme sind auf jeweils einen singulären Zweck optimiert, finden nur regionale Anwendung und decken die bei einem MANV notwendige übergreifende Funktionalität nicht unbedingt ab.

\section{Verbesserungspotenziale und Lösungsansätze}

Entscheidend für alle nachfolgenden Betrachtungen ist, dass ein MANV in den allermeisten Fällen kein Katastrophenfall

Notfall Rettungsmed 2013 · 16:549-555 DOI 10.1007/s10049-013-1764-0

(C) The Authors 2013. This article is published with open access at link.springer.com

\section{A. Donner · C. Adler \\ Patientenregistrierung im Massenanfall. Zukünftige Anforderungen an ein elektronisches Dokumentationssystem}

\section{Zusammenfassung}

Hintergrund. Die effiziente Bewältigung eines Massenanfalls von Verletzten und Erkrankten erfordert sowohl gut abgestimmte und geübte Vorgehensweisen der Einsatzkräfte als auch reibungslosen Informationsaustausch zwischen allen Beteiligten und durch alle Führungsebenen. Bestehende Systeme für die Patientensichtung, -registrierung und Rettungsmitteldisposition basieren auf händisch gepflegten Listen, die sprachlich per Funk und Telefon abgeglichen werden müssen. Elektronische Hilfsmittel können Informationsflüsse deutlich beschleunigen. Aus dem komplexen, nicht planbaren Einsatzgeschehen ergeben sich vielfältige Anforderungen an die Technik und an ein zukünf- tiges elektronisches Dokumentationssystem, die zu berücksichtigen sind und die sich aus der Arbeit von mehreren Forschungsprojekten in den letzten Jahren entwickelt haben. Schlussfolgerung. Eine bundesweite Harmonisierung von Vorgehensweisen und Schnittstellen ist notwendig, sodass reibungslose überregionale Zusammenarbeit möglich ist.

\section{Schlüsselwörter}

Massenanfall von Verletzten und Erkrankten · Sichtungsalgorithmus . Patientenregistrierung · Vorsichtung . Elektronisches Dokumentationssystem

\section{Mass casualty incident patient registration. Electronic documentation requirements}

\section{Abstract}

Background. Efficient handling of mass casualty incidents $(\mathrm{MCl})$ requires well-trained rescue teams and a well prepared course of action. Furthermore, smooth information interchange between all decision makers is mandatory. Existing systems for triage, registration, and management of transport means are based on various lists on paper which have to be synchronized by means of voice and radio systems. Electronic tools can significantly accelerate data flow. Various requirements for a future digital documentation sys- tem arise from the unpredictable conditions in the field. Several projects have researched this topic in recent years.

Conclusion. Nationwide harmonization of procedures and interfaces is necessary for smooth interregional cooperation.

\section{Keywords}

Mass casualty incident - Triage algorithm . Patient logistics · Pre-triage · Electronic documentation im Sinne der Gesetzgebung der Länder ist, sondern eine vorübergehende Überlastung der Rettungsdienste und Krankenhäuser. Eine notwendige bundesweite Harmonisierung steht deshalb nicht im Widerspruch zum Katastrophenschutzauftrag der Länder, wobei allerdings deren Rettungsdienstgesetze zu beachten sind.

In den durchführenden Forschungsprojekten zeigte sich, dass sich der mSTaRT-Algorithmus als Einsatzstandard in den Bundesländern Bayern und Berlin durchsetzt, während beispielsweise in Frankfurt STaRT angewendet wird [8]. Gemeinsamer Nenner ist die algorithmusbasierte Vorgehensweise, die vom Sichtungsteam die konsekutive Abarbeitung einer Reihe von Fragen verlangt.

\section{1) Der Sichtungsvorgang wird in eine Vorsichtung und eine ärztliche (Nach-) Sichtung aufgeteilt}

Bei einem Großschadensereignis wird oftmals davon ausgegangen, dass nicht genügend ärztliches Personal zur Verfügung steht, weswegen der Sichtungsvorgang in eine Vorsichtung, die von ausgebildeten Rettungsassistenten nach dem (m) StaRT-Algorithmus durchgeführt werden kann, und eine ärztliche (Nach-)Sichtung aufgeteilt wird [4]. In der Literatur findet sich ein mittlerer Zeitbedarf für die Vorsichtung durch Rettungsassistenten nach 


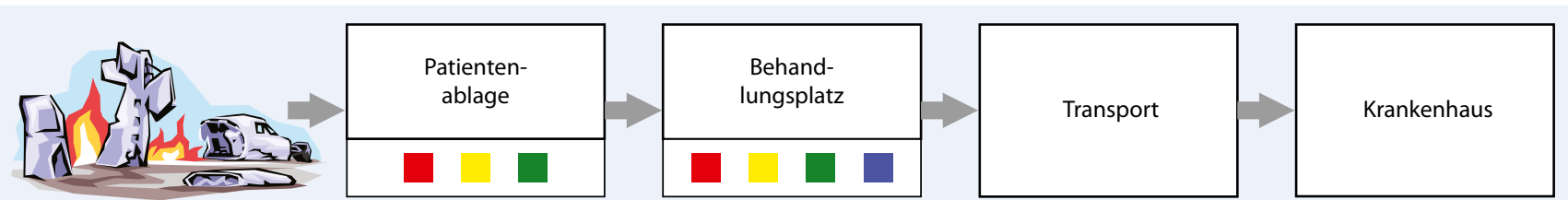

ID, Sichtungskategorie

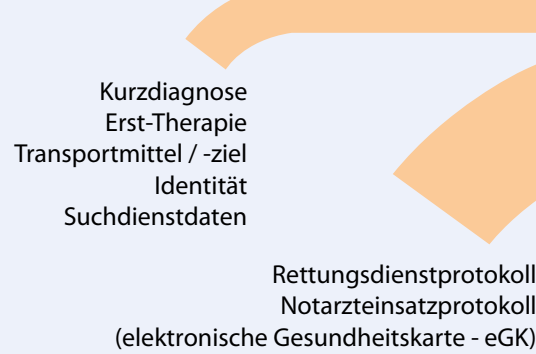

Krankenhausinformationssystem

Abb. $2 \Delta$ Patientendaten entlang der Versorgungskette. (Mit freundl. Genehmigung von Donner et al. [12])

mSTaRT im Rahmen von Übungen bis zum Setzen der Farbkodierung der Patientenanhängekarte von $35 \mathrm{~s}$ je Notfallpatient [9].

Die Testung des e-Triage-Demonstrators bei 29 Einsatzkräften, die im Rahmen einer Großveranstaltung mit Hilfe von $\mathrm{Pa}$ tientenkarten nach einer Kurzeinweisung auf das Gerät tätig waren, ergibt einen Mittelwert von 26,1 s für den mSTaRTAlgorithmus ohne Scannen/endgültiges Speichern. In einem vorhergehenden Labortest von zwölf Teams mit der allerersten Version der Benutzungsoberfläche unter Stressbedingungen finden wir einen Mittelwert von 52 s für die Durchführung der algorithmusbasierten Sichtung inklusive Scanvorgang. Bei 49 durchgeführten Messungen im Rahmen einer abschließenden Testung unter Nutzung von Patientenkarten benötigten die Sichtungsteams für die Vorsichtung und Registrierung im Mittel 46,2 s. Davon wurden 26,3 s für die Vorsichtung (mSTaRT) und 19,9 s für die Registrierung benötigt. In der Überprüfung der Vorsichtungsergebnisse auf Korrektheit wurden bei Erprobung E2 81,2\% korrekt gesichtet (im Vergleich: ohne Gerät 66,7\%). Die spätere Testung Erprobung E3 ergibt einen fast gleichen Wert von 81,6\% [10].

Im Projekt SOGRO betrug die durchschnittliche Zeit 53 s bei 30 beobachteten Vorgängen in einer Übung. Insgesamt wurden von 520 Verletzten $81,5 \%$ richtig eingestuft [8]. Bei A.L.A.R.M-Projekt-
Übungen dauerte die Vorsichtung im Median 45 s bei 33 Patienten.

\section{Jederzeit aktuelles Lagebild \\ - Vorteil beim Einsatz \\ elektronischer Hilfsmittel bei \\ Vorsichtung und Registrierung}

Entscheidender Vorteil beim Einsatz von elektronischen Hilfsmitteln bei der Vorsichtung und Registrierung ist in jedem Fall die nahezu zeitgleiche (d. h. nach jedem gesichteten Patienten) Aktualisierung eines Lagebilds für die Einsatzleitung und die damit verbundenen schnelleren strategischen Entscheidungsmöglichkeiten auf der Führungsebene, die in früheren Studien nicht berücksichtigt und gemessen wurden. Dieser systematische und systemweite Vorteil gegenüber der heutigen papier- und sprachbasierten Vorgehensweise kann als Kriterium für die Performanz der Gesamtlösung nicht genügend betont werden.

\section{Sichtung unter stressreichen Arbeitsbedingungen}

Da die mit der Sichtung betrauten Einsatzkräfte bei einem MANV unter großem Arbeitsdruck stehen und vielfältigen Stressoren ausgesetzt sind, können sie auf ihr Arbeitsgedächtnis, ihr Wissen und ihre Erfahrung nicht in dem üblichen Maß zugreifen [10]. Durch die von einer graphischen Benutzungsoberfläche vorgegebene algorithmusbasierte Abfrage werden Rettungskräfte (inklusive Ärzte) unterstützt, schneller in ihren Arbeitsrhythmus zu kommen und auf ihr Wissen zuzugreifen. Die Sichtungszeiten verringern sich vor allem bei den Personen, die sich beim papierbezogenen Verfahren unsicher sind und den Algorithmus nicht vollständig einhalten. Ein Abweichen vom Algorithmus, das in der Regel zu Störungen im Arbeitsablauf führt, wird verhindert.

\section{Geräteeinsatz und Datenerhebung}

Der Einsatz von elektronischen Datenverarbeitungssystemen kann wesentlich zur effizienten Bewältigung eines MANV beitragen, allerdings dürfen technische Lösungen nicht exklusiv für den Einsatz in dieser Ausnahmesituation geschaffen werden, sondern müssen eine „natürliche“ Erweiterung eines bei der täglichen Arbeit eingesetzten Systems darstellen. Einzusetzende Geräte müssen in den Rettungswagen mitgeführt werden, da diese Fahrzeuge immer frühzeitig am Einsatzort eintreffen. ${ }^{3}$ Und nur wenn die Technik in der täglichen Rettungsdienstarbeit sinnvoll eingesetzt werden kann, sind sowohl die Wirtschaftlichkeit der Anschaf-

\footnotetext{
3 Sanitätsabsicherungen bei Großveranstaltungen sollten ebenfalls entsprechend geschult und technisch ausgestattet sein.
} 
fung und der geübte Umgang gewährleistet.

Erstes Ziel eines umfassenden technischen Ansatzes muss sein, dass die Vorsichtung menügeführt und die Registrierung der Patienten und Betroffenen elektronisch erfolgt. Auf diese Weise können die verteilt erhobenen Daten im Idealfall bereits direkt nach ihrer Erfassung den Entscheidungsträgern zur Verfügung gestellt werden. Ein beispielhaftes Vorgehen für die Vorsichtung wäre wie folgt:

1. Ein Sichtungsteam wird von der Benutzungsoberfläche des Erfassungsgeräts durch einen Sichtungsalgorithmus geführt. Des Weiteren können zusätzliche Daten wie z. B. Minimaldiagnose, Fundort usw. erhoben oder automatisiert erfasst werden.

2. Entsprechend der Sichtungskategorie wird der/die Betroffene mit einer maschinenidentifizierbaren/maschinenlesbaren elektronischen Patientenanhängekarte (ePAK) versehen, die die festgestellte Sichtungskategorie symbolisiert (z. B. Farbgebung und Ziffer). Im einfachsten Fall könnte dies auch lediglich ein Armband mit maschinenlesbarer ID sein.

3. Die erhobenen Daten werden im Gerät gespeichert und bei Verfügbarkeit eines drahtlosen Datennetzes zu einem Serversystem übertragen, das physikalisch vor Ort und/oder im Internet steht. Zudem können die Daten auf der Anhängekarte gespeichert werden (z. B. mittels eines beschreibbaren RFID-Transponders).

4. Technische Lösungen unterstützen die sog. „Umsichtung“ und sorgen für eine Aktualisierung des Gesamtbilds.

Entlang der weiteren Stationen der Versorgungskette können, wie in $\bullet$ Abb. 2 dargestellt, weitere Daten ergänzt werden.

》) Die verteilt erhobenen

Daten sind nahezu in

Echtzeit für die involvierten Entscheidungsträger verfügbar

Hauptvorteil der digitalen Erfassung der Patienten ist, dass die verteilt erhobenen Daten problemlos dupliziert und nahezu in Echtzeit den involvierten Entschei- dungsträgern zur Verfügung gestellt werden können. Um einen echten Mehrwert gegenüber existierenden papier-/sprachbasierten Ansätzen zu erreichen, ist es allerdings notwendig, dass Informationen nicht nur vom, sondern auch zum Einsatzgeschehen bzw. innerhalb dessen übertragen werden können.

Die Zuordnung eines Patienten zu einem Transportmittel zu einem aufnehmenden Krankenhaus involviert Einsatzleitung (Leitender Notarzt, Organisatorischer Leiter, Transportkoordinatoren, Einsatzabschnittsleiter), Leitstelle und Krankenhaus. Einsatzleitung/Transportkoordinatoren entscheiden, welcher Patient mit welcher Dringlichkeit in welches Zielkrankenhaus gebracht wird. Gleichzeitig muss von den Krankenhäusern zurückgemeldet werden, welche Aufnahmekapazitäten noch zur Verfügung stehen. Die Leitstelle koordiniert schließlich entsprechend der Transportanforderungen die Fahrzeuge.

\section{Sprache ist und bleibt das primäre Kommunikations- medium im Einsatzgeschehen}

Sprache sollte nur für zeitkritischen Informationsaustausch eingesetzt werden, nicht aber für die Patientenverwaltung. Erfahrungsgemäß sind die verfügbaren Sprechfunkkanäle durch die Koordinierung der eintreffenden Rettungskräfte stark ausgelastet. Digitale Datenübertragung ist für die Patientenlogistik wesentlich besser geeignet, erfordert aber auch entsprechende Kommunikationsinfrastruktur. Von kommerziellen Mobilfunknetzen ist abzuraten, da mit diesen Systemen nur sehr schlecht Vorrangschaltungen für BOS-Nutzer geschaffen werden können, sodass insbesondere bei Massenveranstaltungen mit Überlastungen dieser Netze zu rechnen ist.

Das im Aufbau befindliche digitale BOS-Netz ist prinzipiell als Datennetz für die Patientendisposition verwendbar, da es anders als die kommerziellen Mobilfunknetze nur einem begrenzten Nutzerkreis zur Verfügung steht und hochverfügbar ausgelegt ist. Wegen sicherheitstechnischer Überlegungen ist jedoch davon auszugehen, dass nichtpolizeilichen Organisationen die Datenübertragung gar nicht oder nur in sehr beschränktem Ausmaß gestattet wird. Zudem sind die zur Verfügung stehenden Datenübertragungsraten technikbedingt sehr begrenzt und werden insbesondere bei Großeinsätzen von polizeilichen und nichtpolizeilichen Kräften gemeinsam verwendet.

Komponenten für IP-basierte Drahtlosnetze („wireless local area network“, WLAN) sind standardisiert, kostengünstig und bei der nichtpolizeilichen Gefahrenabwehr bereits weit verbreitet. WLAN hat zwei wesentliche Nachteile:

- Jeder eingesetzte WLAN-Router benötigt eine eigene Verbindung zum Internet (drahtgebunden oder drahtlos).

- Die maximale zugelassene effektive isotrope Strahlungsleistung der Sendeantennen ist regulatorisch begrenzt, was unter idealen Bedingungen eine Reichweite in der Größenordnung von bestenfalls wenigen $100 \mathrm{~m}$ erlaubt. Repeater sind eine Möglichkeit, die Reichweiteneinschränkung einzelner WLAN-Router zu umgehen, allerdings ist diese Technik nicht sehr effizient und löst auch das Problem nicht, die WLAN-Router von zwei oder mehr unterschiedlichen Organisationen am Einsatzort direkt miteinander zu verbinden.

Eine elegante Lösung zeichnet sich mittlerweile in Form des neuen IEEE 802.11-Standards ab, der im Mai 2012 veröffentlicht wurde und vermaschte Netzstrukturen mit entsprechend größerer Flächenabdeckung und lediglich einer Verbindung zum Internet erlaubt.

In jedem Fall muss ein elektronisches System für die Patientenerfassung auch ohne Verbindung zum Internet funktionsfähig sein. Alternativ kann die Verbindung vom Einsatzort zum Internet und somit zu einer Leitstelle auch mittels Satellitenkommunikation aufgebaut werden. Hierbei ist zu beachten, dass einzusetzende Software mit der beträchtlich längeren Signallaufzeit umgehen kann

Auch aufgrund der Verfügbarkeit von leistungsfähiger und dennoch kostengünstiger Hardware (Notebook, TabletPC, Smartphone) für die Patientenerfassung beschäftigen sich derzeit sehr viele Rettungsorganisationen mit Verbesserun- 
gen in diesem Bereich. Die Forschungsprojekte A.L.A.R.M., e-Triage, MANET, SOGRO und UCSE haben folgende gemeinsame Empfehlungen unter Moderation von e-Triage entwickelt:

\section{Homogenisierung und \\ Zusammenführung der bestehenden Systeme in ein integriertes Gesamtsystem}

Angestrebtes Ziel ist die Homogenisierung und Zusammenführung der bestehenden Systeme in ein integriertes Gesamtsystem. Die Kompatibilität von Lösungen muss auf vielen Ebenen erreicht werden. Die wichtigsten Aspekte sind wie folgt:

1. Bundesweit einheitliches verpflichtendes Datenschutzkonzept für die in einem MANV (und im Rettungs-/ Notarztdienst) erhobenen Patientendaten. Bei einem MANV könnte im einfachsten Fall mit anonymen IDs gearbeitet werden, was den Datenschutz deutlich erleichtert.

2. Harmonisierung der verwendeten Vorsichtungsalgorithmen und daraus resultierender Sichtungskategorien. Festlegung eines einheitlichen Standards für alle Bundesländer.

3. Harmonisierung von Patientenanhängekarten (wie bereits 2004 vom Ausschuss Rettungswesen gefordert [4]). Mit einem Stift beschreibbare Felder sollten trotz elektronischer Systeme weiterhin als Rückfallebene vorgehalten werden. Entscheidend ist dabei nicht die äußere Form der Karte (z. B. Armband oder Anhängekarte), sondern die auszufüllenden Felder und die einzustellende Sichtungskategorie.

4. Einheitliches und eindeutiges Nummerierungssystem für ePAKs, damit ein elektronisches System überhaupt eingesetzt werden kann.

5. Standardisierte Datenfelder und -formate bzw. Schnittstellen. Eine definierte Schnittstelle erleichtert auch die nachfolgenden polizeilichen Ermittlungsarbeiten, die psychosoziale Versorgung, die Gesundheitsfürsorge und die Angehörigenzusammenführung.
6. Verwendung des elektronischen Heilberufsausweises für die Authentifizierung des Anwenders am Gerät, die Verschlüsselung und elektronische Signatur.

7. Einheitliche Formate für die elektronische Lesbarkeit (und Beschreibbarkeit) von ePAKs. Im einfachsten Fall wird lediglich die eindeutige Nummer der Karte ausgelesen, im erweiterten Fall werden Patientendaten elektronisch auf der Karte abgelegt, sodass sie sowohl am Patienten verbleiben als auch an einen Rechner bei der Einsatzleitung übertragen werden. Mischformen müssen ebenfalls möglich sein, d. h. auch ohne spezielle Lesegeräte müssen autorisierte Anwender Zugriff auf die Daten bekommen können. Beispielsweise sollte durch Abtippen der eindeutigen ID der gewünschte Patientendatensatz nachgeschlagen werden können.

8. In Echtzeit elektronisch verfügbarer Versorgungskapazitätsnachweis der Krankenhäuser am Einsatzort und in der Leitstelle [11].

9. Festlegung geeigneter Gestaltungsgrundsätze und Interaktionskonzepte für den Einsatz mobiler Technologien in sicherheits- und zeitkritischen Kontexten.

Fernziel muss sein, dass eine Rettungsdienstorganisation eine beliebige Hardware- und Softwarekombination (für $\mathrm{Pa}$ tientenerfassung, Lagedarstellung, Kräftemanagement, Krankenhauszuweisung, interdisziplinärem Versorgungsnachweis ${ }^{4}$ usw.) von beliebigen Herstellern auswählen kann, die den individuellen Bedürfnissen entspricht, aber dennoch kompatible Schnittstellen zu den anderen am Markt erhältlichen Produkten hat.

Bereits jetzt sind viele Einsatzleitfahrzeuge von verschiedenen Organisationen mit WLAN-Technik ausgestattet, was aber nicht notwendigerweise bedeutet, dass WLAN-fähige Geräte in organisationsfremden Funkzellen betrieben wer-

\footnotetext{
${ }^{4}$ Der webbasierte interdisziplinäre Versorgungsnachweis (IVENA) ist in vielen Städten bereits eingeführt bzw. in Vorbereitung (z. B. flächendeckend in Hessen, München). Ein MANVModul wurde in Frankfurt nachträglich implementiert.
}

den können. Hier besteht wesentlicher organisatorischer Abstimmungsbedarf. Im Gegensatz zu IT-Lösungen für den Heimund Bürobereich müssen Anwendungssoftware und Netzwerkarchitektur integriert betrachtet werden, um die notwendige Robustheit zu erreichen [12].

\section{Fazit für die Praxis}

- Die elektronische algorithmusbasierte Patientenregistrierung darf thematisch nicht nur auf die (Vor-)Sichtung beschränkt werden, sondern muss als komplexes übergreifendes Problem verstanden werden.

- Patienten- und Betroffenenregistrierung und -disposition schließen immer die komplette Versorgungskette vom Schadensort bis zum Krankenhaus ein und involvieren die Entscheidungsträger vor Ort und in rückwärtigen Führungseinrichtungen sowie die aufnehmenden Krankenhäuser.

- Das IVENA-System ist ein wichtiger und richtiger erster Schritt. Elektronische Patientenregistrierung wird sich allerdings nur dann einführen lassen, wenn ein tragfähiger Konsens über den zu verwendenden Sichtungsalgorithmus und die Patientenanhängekarte erzielt wurde.

- Bei Großereignissen treffen fast immer Einsatzkräfte von verschiedenen Organisationen aufeinander, die noch nie gemeinsam in Übungen entsprechende Szenarien bearbeiten konnten. Jede Inkonsistenz bei den Vorgehensweisen und dem Informationsaustausch kann die Gesamteffizienz der Einsatzbewältigung deutlich verringern.

- Die notwendigen Geräte sollen im täglichen Betrieb eingesetzt werden können. Ob damit ein Flottenmanagement realisiert wird und/oder das Rettungsdienst-/Notarzteinsatzprotokoll elektronisch erfasst wird, sollte der jeweiligen Organisation überlassen werden. Entscheidend ist die Systemkompatibilität hinsichtlich eines überregionalen Einsatzgeschehens. 


\section{Korrespondenzadresse}

\section{A. Donner}

Deutsches Zentrum für Luft- und Raumfahrt e.V., Institut für Kommunikation und Navigation, Oberpfaffenhofen

82234 Weßling

anton.donner@dlr.de

Danksagung. Die Autoren bedanken sich bei den folgenden Personen für die wertvollen Diskussionen, Beiträge, Anregungen und Korrekturen:

- Dr. Holger Kirchner, Dr. Martin Dotzer, Georg Rötzer; Fachberater e-Triage

- Stephan Düsterwald, Dr. Torsten Schröder, Dr. Martin Schultz; Charité Berlin

- Mario Di Gennaro, Prof. Leo Latasch; DRK Frankfurt

- Reiner Arlt, Michael Ben-Amar, Thomas GreinerMai; Euro-DMS Ltd

- Dr. Marion Krüsmann, Anton Metz, Lena Erfurt; Dept. Psychologie, Ludwig-Maximilians-Universität München

- Dr. Markus Werner; TriaGnoSys GmbH

- Prof. Martin C. Kindsmüller; Universität Hamburg, Fachbereich Informatik, Mensch-Computer-Interaktion

- Prof. Dr. Michael Herczeg, Tilo Mentler; Universität zu Lübeck, Institut für Multimediale und Interaktive Systeme

- Nils Ellebrecht, Markus Jenki, Prof. Dr. Stefan Kaufmann; Institut für Soziologie, Albert-Ludwigs-Universität Freiburg

- Benedikt Weber; antwortING Ingenieurbüro, Köln

\section{Einhaltung der ethischen Richtlinien}

Interessenkonflikt. A. Donner und C. Adler waren am Forschungsprojekt e-Triage beteiligt. Dieser Beitrag beinhaltet keine Studien an Menschen oder Tieren.

\section{Open Access}

This article is distributed under the terms of the Creative Commons Attribution License which permits any use, distribution, and reproduction in any medium, provided the original author(s) and the source are credited.

\section{Literatur}

1. DIN 13050. Rettungswesen - Begriffe. Normenausschuss Rettungsdienst und Krankenhaus (NARK) im DIN und Normenausschuss Feuerwehrwesen (FNFW) im DIN (2009) Vertrieb durch Beuth Verlag $\mathrm{GmbH}, 10772$ Berlin

2. Ellebrecht N (2009) Triage. Charakteristika und Gegenwart eines ordnungsstiftenden Verfahrens. Sociol International 47:229-257

3. Adler C, Donner A, Kühling M, Krüsmann M (2011) (Vor-)Sichtung und elektronische Betroffenenerfassung bei einem Massenanfall von Verletzten. In: Mendel K, Hennes P (Hrsg) Handbuch des Rettungswesens, A 1.4 [20]. Mendel, Witten. ISBN 9783-930670-30-7
4. Gräff G (2007) Bericht der Arbeitsgruppe „Massenanfall von Verletzten und Erkrankten - MANV “ des Ausschusses „Rettungswesen". Ständige Konferenz der Innenminister und -senatoren der Länder, Arbeitskreis V, Ausschuss Rettungswesen. http:// www.stmi.bayern.de/imperia/md/content/stmi/ sicherheit/rettungswesen2/publikationen/ausschussrettungswesen/manv 11 2007.pdf

5. Donner A, Erl S, Adler C et al (2011) Projekt e-Triage: Datenmanagement für einen Massenanfall von Verletzten.: INFORMATIK 2011 - Workshop zur ITUnterstützung von Rettungskräften. Gesellschaft für Informatik e. V., Berlin

6. Sefrin P, Weidringer JW, Weiss W (2002) Dokumentation der Sichtung bei Großschadensereignissen und Katastrophen. Bericht zur 2. Konsensus-Konferenz in Bad Breisig am 29. Oktober 2002. BVA AKNZ/Bundesvereinigung der Arbeitsgemeinschaften der Notärzte Deutschlands. http://www. agsan.de/scripte/konsenskonf.pdf

7. Messelken M, Schlechtriemen T, Arntz HR et al (2011) Der Minimale Notfalldatensatz MIND3. DIVI 2:130-135. Deutscher Ärzte-Verlag

8. Ellebrecht N, Latasch L (2011) Vorsichtung durch Rettungsassistenten auf der Großübung SOGRO MANV 500. Notfall Rettungsmed 1-6

9. Gutsch W, Huppertz T, Zollner C et al (2006) Initiale Sichtung durch Rettungsassistenten - Ergebnisse bei übungen zum Massenanfall von Verletzten. Notfall Rettungsmed 9:384-388

10. Adler C, Erfurt L, Metz A, Krüsmann M (2012) Schlussbericht e-Triage: Elektronische Betroffenenerfassung in Katastrophenfällen. FKZ 13N10541, Ludwig-Maximilians-Universität, München. http://edok01.tib.uni-hannover.de/edoks/ e01fb13/747134626.pdf

11. Schweigkofler U, Reimertz C, Auhuber T et al (2011) Web-basierter Versorgungskapazitätsnachweis. Unfallchirurg 114:928-937

12. Donner A, Arlt R, Greiner-Mai T (2012) Jenseits von Papier und Sprache. Anmerkungen zur MANV-Bewältigung mit digitaler Informations- und Kommunikationstechnik. In: Bergh B, Rashid A, Röhrig $\mathrm{R}$ (Hrsg) 1. Symposium ICT in der Notfallmedizin (NotIT). German Medical Science, Rauischholzhausen, 73-79. http://www.egms.de/static/en/meetings/notit2012/12notit10.shtml

\section{Hier steht eine Anzeig}

Springer 